\title{
Differentially expressed mRNAs, IncRNAs, and miRNAs with associated co-expression and ceRNA networks in ankylosing spondylitis
}

\author{
Chen Zhang ${ }^{1, *}$, Chen Wang ${ }^{1, *}$, Zhenyu Jia ${ }^{1, *}$, Wenwen Tong ${ }^{1}$, Delin Liu ${ }^{1}$, Chongru \\ $H^{1}{ }^{1}$, Xuan Huang ${ }^{1}$ and Weidong $X u^{1}$ \\ ${ }^{1}$ Department of Orthopedics, Changhai Hospital, Second Military Medical University, Shanghai, China \\ *These authors contributed equally to this work \\ Correspondence to: Weidong Xu, email: xuwdshanghaichyy@126.com \\ Xuan Huang, email: 11791709@qq.com
}

Keywords: ankylosing spondylitis; microarray; competing endogenous RNA; long noncoding RNA; miRNA

Received: September 21, $2017 \quad$ Accepted: October 29, $2017 \quad$ Published: November 27, 2017

Copyright: Zhang et al. This is an open-access article distributed under the terms of the Creative Commons Attribution License 3.0 (CC BY 3.0), which permits unrestricted use, distribution, and reproduction in any medium, provided the original author and source are credited.

\section{ABSTRACT}

Ankylosing spondylitis (AS) is a chronic autoimmune disease characterized by systemic inflammation and pathological osteogenesis. However, the genetic etiology of AS remains largely unknown. This study aimed to explore the potential role of coding and noncoding genes in the genetic mechanism of AS. Using microarray analyses, this study comprehensively compared IncRNA, microRNA, and mRNA profiles in hip joint ligament tissues from patients with AS and controls. A total of 661 IncRNAs, 574 mRNAs, and 22 microRNAs were differentially expressed in patients with AS compared with controls. Twenty-two of these genes were then validated using realtime polymerase chain reaction. Gene ontology and pathway analyses were performed to explore the principal functions of differentially expressed genes. The pathways were involved mainly in immune regulation, intercellular signaling, osteogenic differentiation, protein synthesis, and degradation. Gene signal transduction network, coding-noncoding co-expression network, and competing endogenous RNA expression network were constructed using bioinformatics methods. Then, two miRNAs, miR-17$5 p$ and $m i R-27 b-3 p$, that could increase the osteogenic differentiation potentials of ligament fibroblasts were identified. Finally, differentially expressed, five IncRNAs, four miRNAs, and five mRNAs were validated using quantitative real-time polymerase chain reaction. These results suggested that mRNAs, IncRNAs, and microRNAs were involved in AS pathogenesis. The findings might help characterize the pathogenesis of AS and provide novel therapeutic targets for patients with AS in the future.

\section{INTRODUCTION}

Ankylosing spondylitis (AS) is a chronic autoimmune disease characterized by systemic inflammation and pathological osteogenesis [1]. It initially involves the sacroiliac joints, and affects mainly the axial skeleton, tendon attachment points, and ligaments [2]. AS eventually leads to ligamentous ossifications and spinal fusion, which bring a great burden to patients and society. Several studies have explored the pathogenesis of inflammation in AS. It has been demonstrated to involve bacterial infection [3], macrophage activation [4], certain cytokines [5-9], HLA-B27 misfolding [10], and autophagy $[11,12]$. However, the real cause of the disease is still unknown. Also, the mechanism of new bone formation remains poorly understood. Therefore, in-depth studies on the precise mechanisms of inflammation and new bone formation in AS should provide valuable information.

Ligaments are band of tissues that hold the ends of bones together at a joint, allowing most joints to move and stabilizing them. They are hypothesized to be the primary target tissue for inflammation and ossification in AS, and are responsible for many of the symptoms in patients with AS $[13,14]$. A previous study has identified alterations in 
the ligaments of patients with AS, including calcification of tissue, significant increases in microvascular density, and irregular arrangements of collagen fibrils [15]. Fibroblasts are the most common cells of ligaments around joints. Recently, some studies demonstrated that fibroblasts contributed to pathological osteogenesis, partly because they expressed osteogenesis-associated genes [16] and had the osteogenic potential to be induced to osteoblasts [17]. Recently, the osteogenic potential of fibroblasts was demonstrated to be greater in patients with AS than in patients with osteoarthritis (OA) [18]. However, the specific molecular changes in ligaments from patients with AS remain to be elucidated.

Over the past decades, noncoding RNAs (ncRNAs), including long ncRNAs (lncRNAs) and short ncRNAs, have gained attention. MicroRNAs (miRNAs) are small noncoding RNAs ( $\sim 22$ nucleotides long) that mostly downregulate the expression of target genes at the posttranscriptional level [19]. MiR-10b-5p was found to be a novel T helper (Th) 17 regulator present in Th17 cells from AS [20]. In addition, serum miR-146a, miR-29a, and miR-155 were significantly upregulated in patients with AS [21, 22]. Dysregulation of these miRNAs in the circulation could be potential biomarkers for AS. However, no studies have focused on miRNA profiles of diseased tissues. LncRNAs are a class of noncoding RNAs more than 200 nucleotides long [23]. Many studies have demonstrated the involvement of lncRNAs in inflammation and osteogenesis. Altered expression of lncRNAs has been implicated in the pathogenesis of different forms of arthritis, including rheumatoid arthritis (RA) [24] and OA [25]. However, the particular functions of IncRNAs in the pathogenesis of AS remain unclear. Recently, the competing endogenous RNA (ceRNA) hypothesis was proposed according to which lncRNAs and other RNA molecules harboring miRNA response elements could compete with one another for binding to a common miRNA, thus regulating miRNA-mediated gene silencing [26]. The involvement of ceRNA network has been demonstrated in several diseases [27-31], but it has not yet been explored in AS.

In the present study, a microarray analysis was performed to compare the hip joint ligament tissues of patients with AS (the AS group) and patients with femoral neck fracture (the control group), and to identify differentially expressed (DE) lncRNAs, miRNAs, and mRNAs. Gene Ontology (GO) categories and Kyoto Encyclopedia of Genes and Genomes (KEGG) pathway enrichment analyses were used to explore specific functions of lncRNAs, miRNAs, and mRNAs. A gene-gene interaction network and lncRNA-mRNA co-expression networks were constructed. The ceRNA regulation network in AS was systematically investigated through co-expression and miRNA-binding prediction analyses. Finally, two DE miRNAs were found to be involved in osteogenic differentiation of ligament fibroblasts. The findings offered greater insights into the pathogenesis of AS and potentially provided new therapeutic targets.

\section{RESULTS}

\section{DE IncRNAs, mRNAs, and miRNAs}

A total of 661 lncRNAs, 574 mRNAs, and 22 miRNAs were found to be DE in the AS group compared with the control group. Of these, 240 mRNAs, 6 miRNAs, and $196 \operatorname{lncRNAs}$ were overexpressed and 334 mRNAs, 16 miRNAs, and 465 lncRNAs were downexpressed in patients with AS compared with controls. The hierarchical clustering analysis of these RNAs is presented in Figure 1A, which shows that the three kinds of RNAs could well distinguish patients with AS from the controls. Finally, volcano plots were constructed to demonstrate differences between the two groups (Figure 1B).

\section{GO and KEGG enrichment analysis of DE miRNA target genes and DE mRNAs}

Target prediction of DE miRNAs was carried out using two online software programs, miRanda and TargetScan. The results of the two target prediction programs were integrated, and only the intersection was selected to increase the specificity. As a result, 3651 target genes were predicted for 16 miRNAs (Figure 2A). Then, GO analyses were performed on the DE miRNA target genes and DE mRNAs. The upregulated and downregulated genes were individually analyzed. The results showed that 166 and 123 GO functions were annotated for upregulated and downregulated miRNA targets. The top 10 statistically significant GO terms are shown in Figure 2B. The results showed that 255 and $247 \mathrm{GO}$ functions were annotated for upregulated and downregulated mRNAs. The top 10 statistically significant GO terms are shown in Figure 3A. Next, the DE miRNA target genes and DE mRNAs were assessed with the KEGG database. The pathways are shown in Figures 2C and 3B (DE miRNA target genes and DE mRNAs, respectively). The predicted target genes were involved in adherens junction, hypoxia-inducible factor-1 (HIF-1) signaling pathway, transforming growth factor- $\beta$ (TGF- $\beta$ ) signaling pathway, extracellular matrix-receptor interaction, focal adhesion, and so forth. In addition, the DE mRNAs were also enriched in biological pathways, such as cytokine-cytokine receptor interaction, TGF- $\beta$ signaling pathway, and Hippo signaling pathway.

\section{Identification of miRNA-gene network and miRNA-GO network}

The miRNA-gene network was built to show the interactions of miRNAs and their target genes. The 
genes were identified from the intersection between 2095 putative target genes of these miRNAs and 574 DE mRNAs. As shown in Figure 4, miR-17-5p was found to be more prominent and crucial in the network because its targets were more than 50. Furthermore, the miRNA-GO network was constructed according to the relationships among significant GOs and miRNAs (Figure 5). MiR$17-5 p$ seemed to be the key node in this network, which regulated 87 biological processes.

\section{Signal network and coding-noncoding gene co-expression network}

The gene signal transduction network was established based on the significant GO and pathway analysis to investigate the relationship of DE genes and their potential role in AS (Figure 6). The genes with the highest betweenness centralities $(\geq 0.01)$ are listed in Table 1. ATM (downregulated), $P H G D H$ (upregulated), ITGA2 (downregulated), and TUBA4A (upregulated) were the four key genes identified by the analysis. ITGA2 had the highest degree of connectivity with other genes. However, ATM and PHGDH were the key nodes and had the highest betweenness centralities in the signal-net. Finally, the coding-noncoding gene co-expression (CNC) network was constructed to identify the interactions between lncRNAs and mRNAs. A total of 521 lncRNAs and 328 mRNAs were used to build the CNC network for the AS group (Supplementary Figure 2) and 547 lncRNAs and 323 mRNAs to build the network for the control group (Supplementary Figure 3). According to the Diffk values ( $\mid$ Diffk $\mid \geq 0.75$ ) for the 2 networks, 24 genes, including 17 lncRNAs and $7 \mathrm{mRNAs}$, showed different connectivities between the AS and control groups, indicating that their roles were important in the core pathways of AS (Table 2).

\section{Construction of ceRNA network}

The ceRNA network was constructed based on coexpressed lncRNAs-miRNAs, miRNAs-mRNAs, and
A

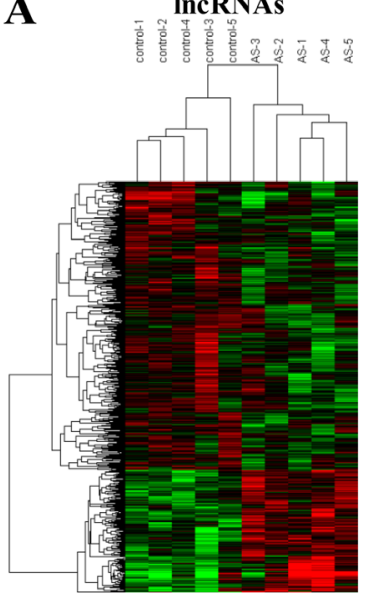

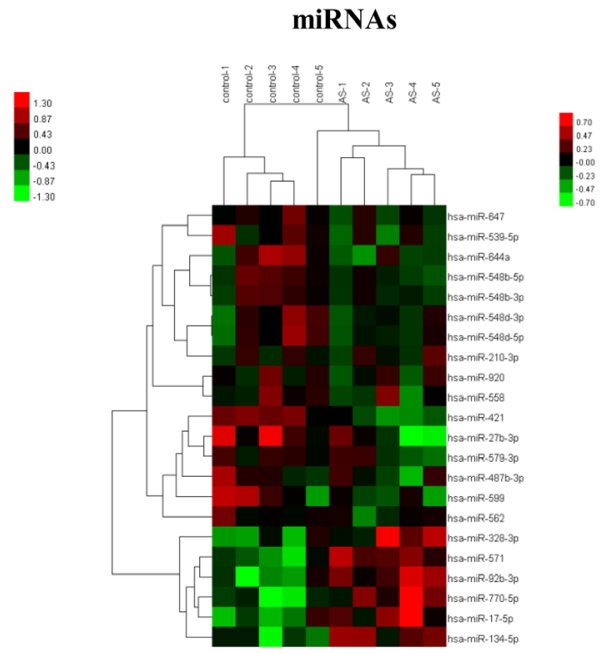
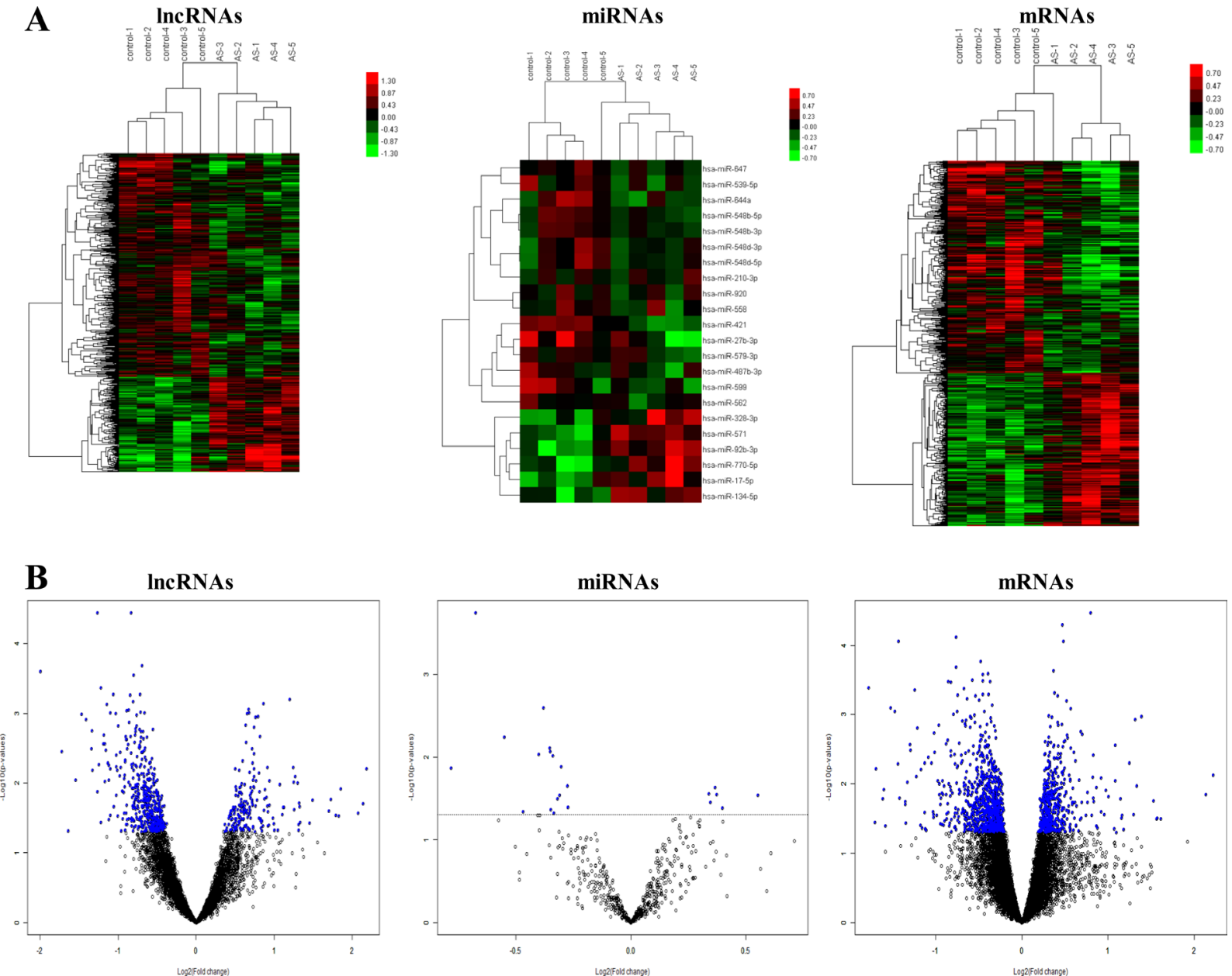

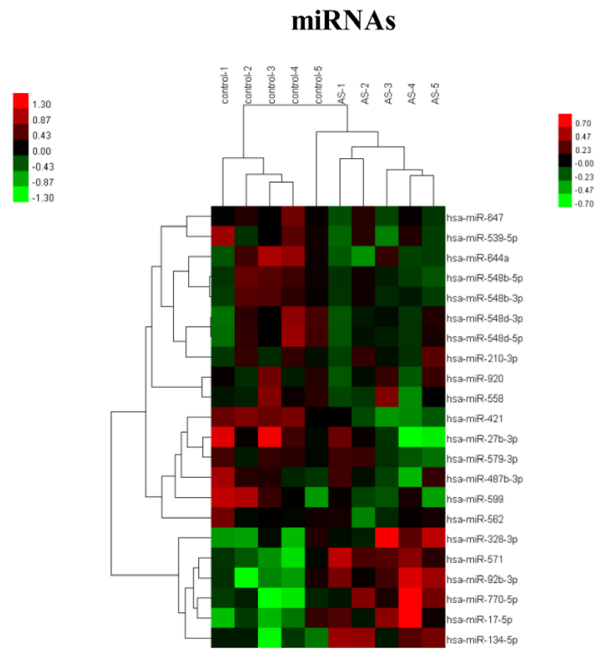

miRNAs

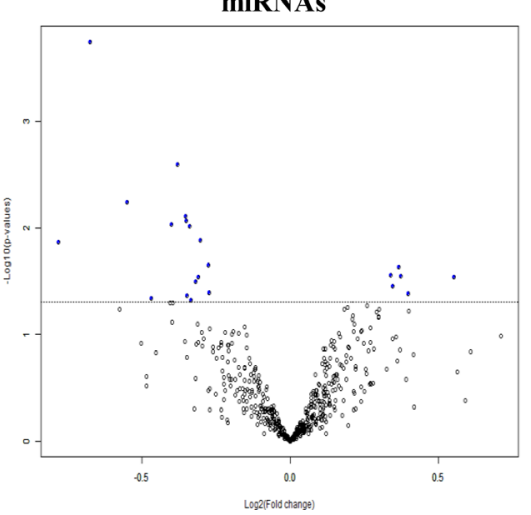

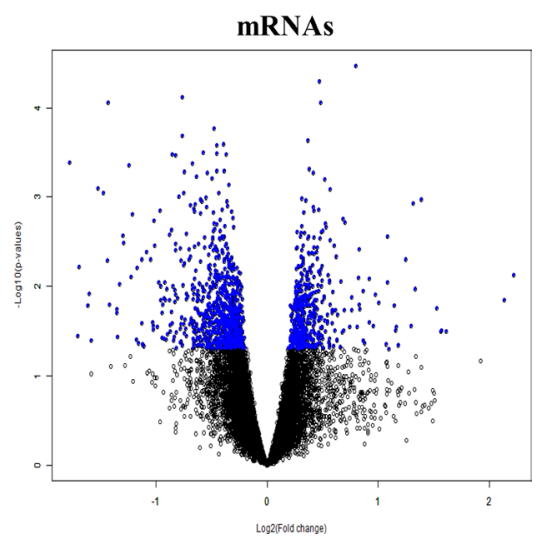

Figure 1: Heatmap and volcano plots showing IncRNA, mRNA, and miRNA levels. (A) Screening criteria were as follows: $P \leq 0.05$ for lncRNAs, miRNAs, and mRNAs. Expression values are depicted in line with the color scale; intensity increases from green to red. Each column represents one sample, and each row indicates a transcript. (B) Volcano plots reflecting number, significance, and reliability of differentially expressed lncRNAs, miRNAs, and mRNAs. The abscissa is $\log 2$ (FC value) and the ordinate is $-\log 10(P$ value). Blue dots are deregulated genes, and black dots are genes that were the same between the two groups. 
lncRNAs-mRNAs. As shown in Figure 7, the lncRNAmiRNA-mRNA network was composed of six lncRNAs, eight mRNAs, and six miRNAs.

\section{Validation of DE IncRNAs, mRNAs, and miRNAs}

Based on the microarray data, five lncRNAs, four miRNAs, and five mRNAs were randomly selected for quantitative real-time polymerase chain reaction (qRT-PCR) verification. DE mRNAs were identified in ligament tissue samples from 15 patients with AS and 24 control subjects. LncRNAs and miRNAs were identified in 15 pairs of tissue samples. LncRNAs NDRG1-AS6 and CSNK1D-AS8 were upregulated, while CD46-AS9, SMYD5-AS2, and NR_045553 were downregulated (Figure 8A). MiR-17-5p and miR-92b-3p were upregulated, while $m i R-27 b-3 p$ and $m i R-539-5 p$ were downregulated (Figure 8B). ANGPT1, PHGDH, ENTPD3, $E P A S 1$, and $I L-33$ were upregulated (Figure $8 \mathrm{C}$ ). The results were consistent with those of the microarray analyses, confirming the reliability of the microarray data (Figure 8D).

\section{MiRNAs were DE during osteogenic differentiation of human ligament fibroblasts}

Human ligament fibroblasts at passage 4 were successfully differentiated into osteoblasts when cultured in osteogenic differentiation medium (OM) for 21 days. The alkaline phosphatase (ALP) activity increased on days 7 and 14 (Figure 9B). A similar result was observed by ALP staining (Figure 9A). Alizarin red S (ARS) staining showed the formation of new calcium nodules on day 21 (Figure 9C). A similar result was observed by quantification of ARS (Figure 9D). Furthermore, the qRT-PCR result showed that ALP, Runx2, and COL1A1 were all significantly upregulated on days 7 and 14 (Figure 9E). Finally, the expression of 6 DE miRNAs was detected during the osteogenic differentiation. The result demonstrated that $m i R-17-5 p$ was upregulated, whereas $m i R-27 b-3 p$ was downregulated (Figure 9F).

\section{DISCUSSION}

AS is the major subtype of spondyloarthritis with an unknown etiology. Thus, understanding the molecular mechanisms of AS would be of immense importance. Previous studies have focused mainly on proteincoding genes [32]. Although the functional relevance of several miRNAs in the plasma of AS has been proved, the involvement of IncRNA in AS is yet to be defined. The lncRNAs, including lnc-ZNF354A-1, lnc-LIN54-1, lnc-USP50-2, and lnc-FRG2C-3, may be involved in the abnormal osteogenic differentiation of mesenchymal stem cells from patients with AS [33]. However, a comprehensive analysis of DE profiles of lncRNAs in AS has not yet been reported. In this study, a microarray was

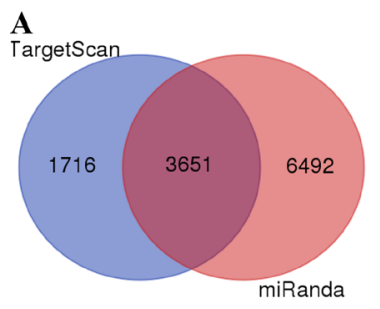

B
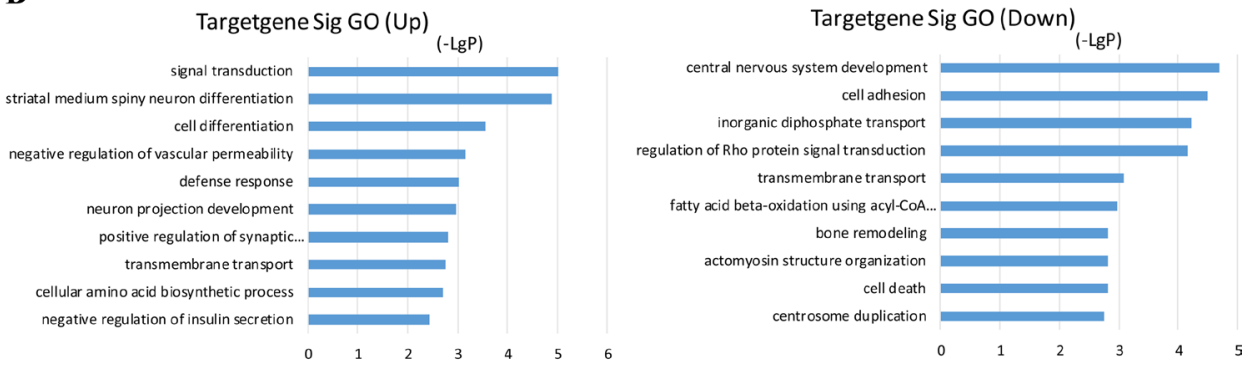

C
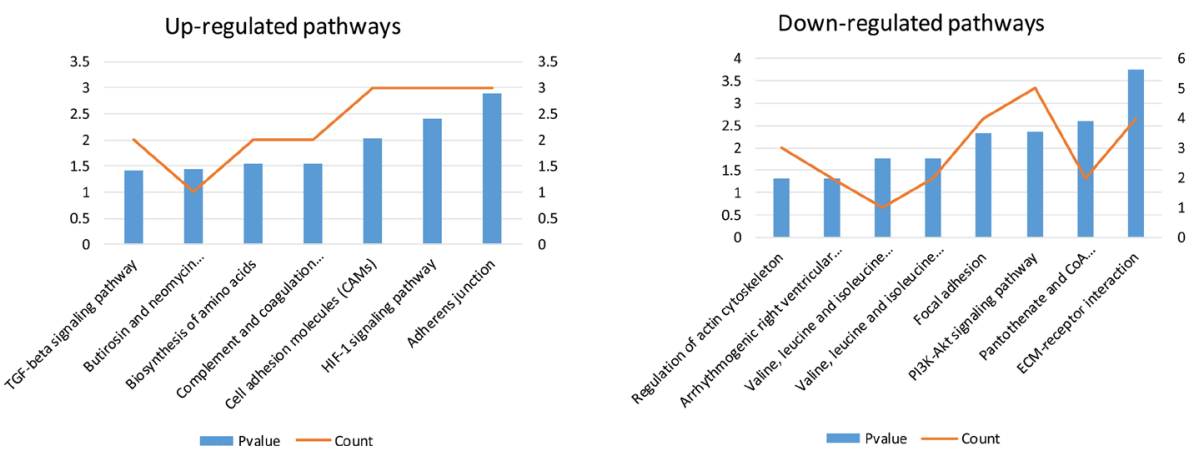

Figure 2: MiRNA target prediction and enrichment analysis of the predicted target genes. (A) Venn diagram of genes targeted by differentially expressed miRNAs. TargetScan and miRanda databases were used to predict miRNA targets. The numbers of predicted target genes were presented in a Venn diagram. (B) GO annotation of upregulated and downregulated mRNAs, with the top 10 functional GO terms. (C) Significant pathways of upregulated and downregulated mRNAs. Enrichment score values were calculated as $-\log 10$ ( $P$ values). GO, Gene Ontology. 
applied to explore the expression profiles of lncRNAs, miRNAs, and mRNAs in patients with AS. This was a novel study to examine DE lncRNAs in AS. Furthermore, a ceRNA network was constructed that provided biological views of lncRNA-miRNA-mRNA interactions. These data might provide strong evidence for the in-depth exploration of the pathogenesis of AS.

The microarray results showed 661 DE lncRNAs, 22 miRNAs, and 574 mRNAs in the AS and control samples. Most of the identified lncRNAs have not been functionally characterized, whereas most of the DE mRNAs and miRNAs are well known. Therefore, bioinformatics analyses of these mRNAs and miRNAs were conducted to help better understand the pathogenesis of AS and speculate on the function of DE IncRNAs. GO and pathway analyses showed that the DE mRNAs and target genes of DE miRNAs were related mainly to inflammation, cell activation processes, angiopoiesis, and ossification that are clearly associated with AS pathogenesis. The KEGG pathway analysis revealed that 39 signaling pathways exhibited significant differences in hip joint ligaments of patients with AS. Based on the analysis, a few AS-related pathways were detected, including cytokine-cytokine receptor interaction, ubiquitin-mediated proteolysis, JAKSTAT signaling pathway, TGF- $\beta$ signaling containing the BMP pathway, PI3K-Akt signaling pathway, Hippo signaling pathway, and HIF-1 signaling pathway. These pathways were involved mainly in immune regulation, intercellular signaling, osteogenic differentiation, and protein synthesis and degradation. Previous studies have demonstrated the involvement of BMP signaling pathway in new bone formation in AS [34]. The PI3K-Akt signaling pathway and its downstream targets were reported to be critical regulators of bone formation and involved in the pathogenesis of AS [35]. The JAK-STAT signaling pathway has also been suggested to regulate Th17 cells in AS [36]. JAK2 polymorphisms have been implicated to be associated with AS [37]. A signal-net analysis was performed on significant GOs and pathways to identify important genes involved in the molecular mechanism of AS. The analysis revealed that ATM and ITGA2 exhibited the highest degrees. ATM was reported to promote abnormal proliferation of breast cancer-associated fibroblasts through maintaining intracellular redox homeostasis and activating the PI3K-Akt, MEK-ERK, and Wnt/ $\beta$-catenin signaling pathways [38]. Moreover, ITGA2 enhanced joint inflammation and cartilage destruction by activating the MEK-ERK signaling pathway [39]. Thus, the signalnet results suggested that these genes were crucial in the pathogenesis of inflammation and ossification in AS.
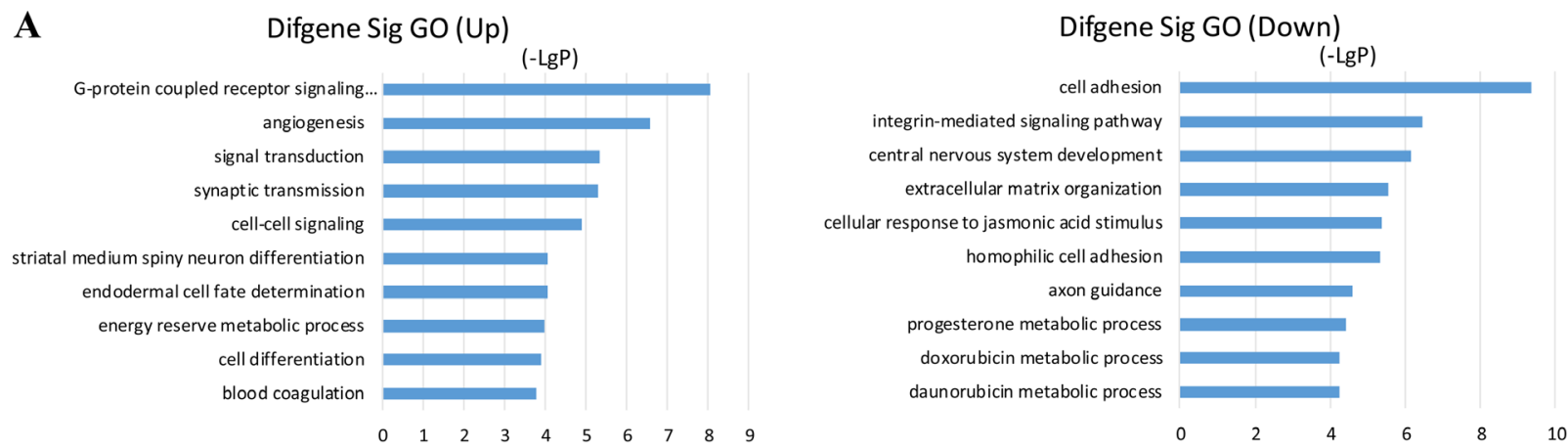

B
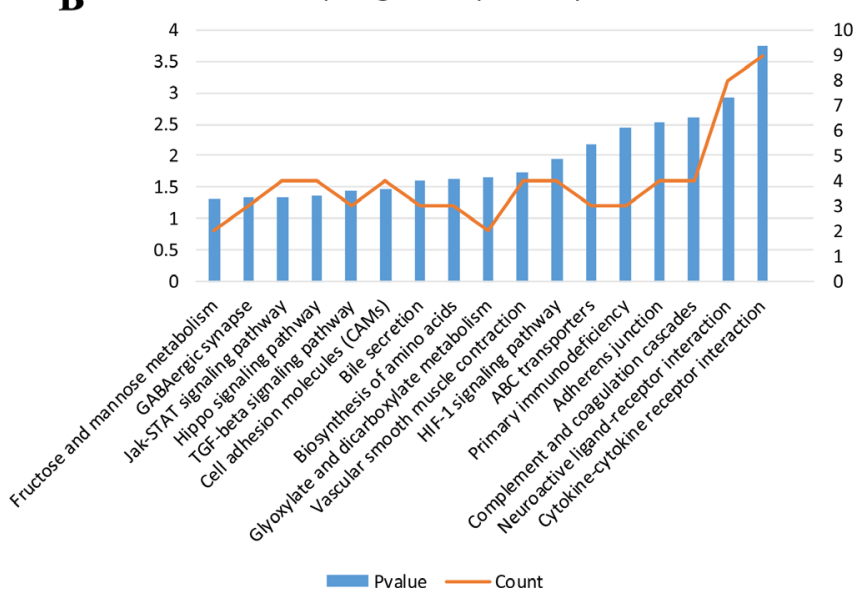

Down-regulated pathways

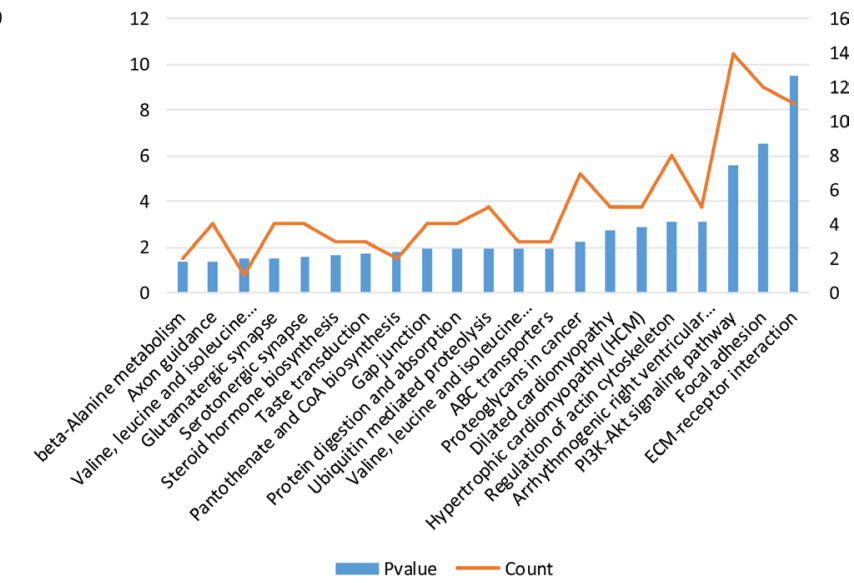

Figure 3: GO and KEGG pathway analysis of the differentially expressed mRNAs. (A) GO annotation of upregulated and downregulated mRNAs, with the top 10 functional GO terms. (B) Significant pathways of upregulated and downregulated mRNAs. Enrichment score values were calculated as $-\log 10$ ( $P$ values). GO, Gene Ontology; KEGG, Kyoto Encyclopedia of Genes and Genomes. 
The CNC network identified some key genes, including VASH1 and SPINK5, and some lncRNAs, including CSNK1D-AS8, NDRG1-AS6, and CD46-AS9. $V A S H 1$, which served as inhibitors of angiogenesis. They were downregulated in hip joint ligaments of patients with AS, thereby participating in the pathogenesis of AS. An imbalance between angiogenic inducers and inhibitors seems to be an important factor in the pathogenesis of autoimmune diseases. Studies have suggested that angiogenesis might be implicated in AS pathogenesis and serve as a therapeutic target [40]. SPINK5 belongs to the protease inhibitor family; it is involved in antiinflammatory and antimicrobial protection of mucous epithelia [41]. CSNK1D-AS8, as a component of Wnt/ $\beta$ catenin and Hedgehog signaling pathways [42], may be implicated in the regulation of cell development and differentiation [43]. Previous studies have reported that NDRG1-AS6 and CD46-AS9 might participate in the pathology of inflammation by regulating the $N F-\kappa B$ signaling pathway $[44,45]$.

In this study, a ceRNA network was constructed based on microarray data. For example, as a ceRNA, lncRNA ENST00000529935 competes for binding to $m i R-548 d-3 p$, thereby affecting the expression of TAP2.TAP2, participating in antigen processing and presentation, was reported to be associated with AS $[46,47]$. Additionally, genetic variations within STXBP6 might influence the response to TNF- $\alpha$ inhibitors in patients with RA [48]. Understanding these novel RNA cross-talks might provide insights into gene regulatory networks with implications in AS.

Recent studies have revealed that several miRNAs might be involved in AS pathogenesis. However, most of the studies were conducted on the plasma. Tissue miRNAs have been noted not only as key molecules in intracellular regulatory networks for gene expression but also as biomarkers for various pathological conditions [49]. This novel study detected miRNAs in tissues of patients with AS. In the present study, several candidate miRNAs were selected to investigate their functions on the osteogenic differentiation of ligament fibroblasts. The experiments showed that $m i R-17-5 p$ was upregulated, whereas $m i R$ $27 b-3 p$ was downregulated. The results were consistent with the microarray data. Previous studies demonstrated that $m i R-17-5 p$ could regulate osteogenic differentiation by targeting several osteogenic markers, such as BMP2, SMAD5, and SMAD7 [50-52]. A recent study showed that $m i R-27 b-3 p$ suppressed osteogenic differentiation of maxillary sinus membrane stem cells by targeting $\mathrm{Sp} 7$ [53]. The findings indicated that $m i R-17-5 p$ and $m i R-$ $27 b-3 p$ were involved in the osteogenic differentiation of ligament fibroblasts. This study hypothesized that these miRNAs might participate in the pathogenesis of new bone formation in AS.

The present study had some limitations. First, the sample size was small. Studies with a large number of patients with AS are needed for obtaining better results. Moreover, the mechanism by which the two miRNAs regulate the osteogenic differentiation of ligament fibroblasts is unknown. Further studies should address these questions.

In summary, the present study provided comprehensive lncRNA, miRNA, and mRNA profiles for AS hip joint ligaments. Bioinformatics approaches were used to predict the potential functions of DE mRNAs and initially explore their roles in the pathogenesis of AS. The cell experiment indicated that miRNAs might
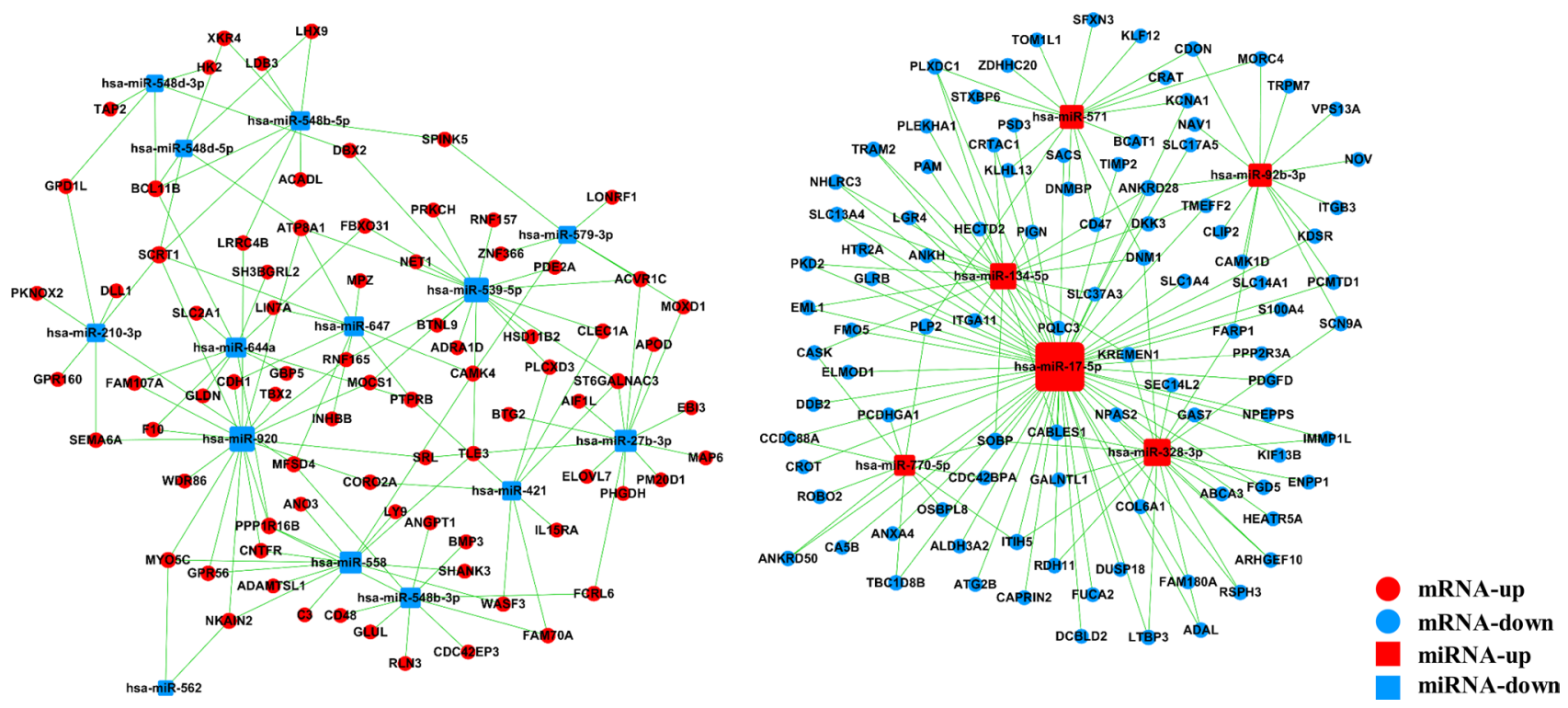

Figure 4: MicroRNA-gene network. Box nodes denote miRNAs (red: upregulated miRNAs; blue: downregulated miRNAs), circle nodes denote genes (red: upregulated genes; purple: downregulated genes), and edges show the inhibitory effect of miRNAs on genes. Degree means the contribution of one miRNA to the genes around or the contribution of one gene to the miRNAs around. The key miRNAs and genes in the network always have the highest degrees. 
participate in ossification. These findings might provide new insights into the pathogenesis of AS and lay a foundation for further functional research. However, the biological functions and specific molecular mechanisms of DE lncRNAs in the pathogenesis of AS warrant further exploration.

\section{MATERIALS AND METHODS}

\section{Patients and samples}

Hip joint ligament tissue samples were obtained from 20 HLA-B27-positive patients with AS undergoing total hip replacement at the Department of Orthopedics in Changhai hospital between January 2013 and December 2016. All of them fulfilled the modified New York criteria for AS [54]. Additional 34 patients with femoral neck fracture who underwent total hip arthroplasty were used as controls. The characteristics of all the patients are shown in Table 3 . The study was approved by the ethics committee of Changhai hospital. Both patients and controls provided written informed consent. All samples were divided into two parts: five pairs of tissues were used for microarray analysis, and the remaining tissues were used for validation by qRT-PCR.

\section{RNA extraction and microarray assay}

The total RNA was extracted from frozen tissues using the RNeasy kit (QIAGEN, Hilden, Germany) according to the manufacturer's instructions. An ND1000 spectrophotometer (NanoDrop Technologies, DE, USA) was used to measure the concentration and purity of RNA. RNA integrity was measured using agarose gel electrophoresis. The microarray experiments were performed using the Affymetrix GeneChip Human Transcriptome Array (Affymetrix, MA, USA). The probes on the array covered all known coding transcripts (including alternative splice variants) and more than 40,000 noncoding transcripts (including lncRNAs, miRNAs, and small nucleolar RNAs). The cDNA labeling, microarray analysis, and bioinformatics analysis were performed by Genminix Informatics (Genminix, Shanghai, China). Briefly, the sample labeling, hybridization, and washing were performed based on the user manual. The arrays were scanned using the GeneChip

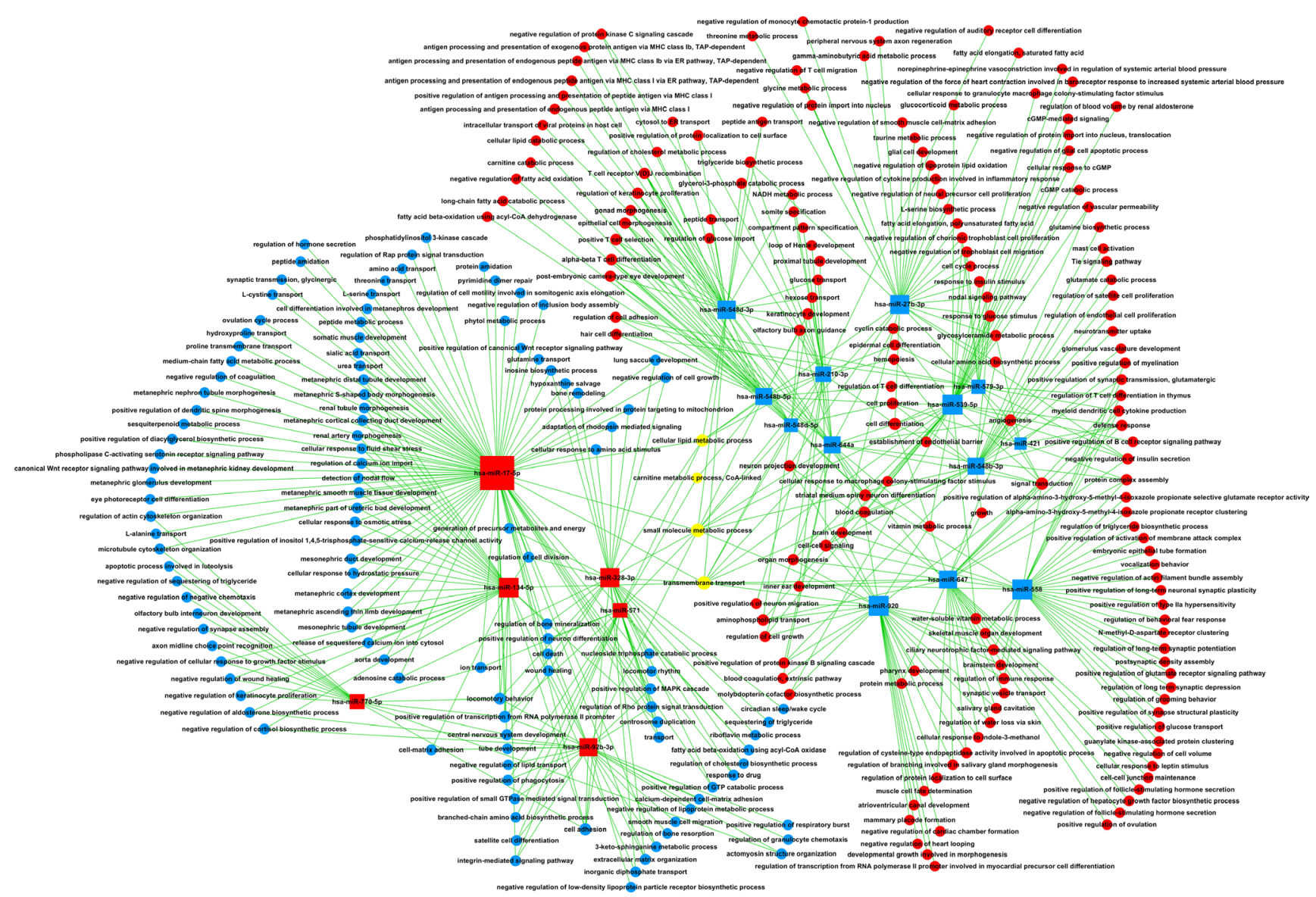

Figure 5: MicroRNA-GO network. Box nodes denote miRNAs (red: upregulated miRNAs; blue: downregulated miRNAs), circle nodes denote GOs (red: upregulated GOs; purple: downregulated GOs), and edges show the inhibitory effect of miRNAs on GOs. Degree means the contribution of one miRNA to the GOs around. The key miRNAs in the network always have the highest degrees. GO, Gene Ontology. 
Table 1: Top genes ranked by betweenness centrality over 0.1 after analysis of signal-net

\begin{tabular}{llccc}
\hline Gene symbol & Description & $\begin{array}{c}\text { Betweenness } \\
\text { centrality }\end{array}$ & Degree & style \\
\hline ATM & Ataxia telangiectasia mutated & 0.0921211 & 16 & Down \\
PHGDH & Phosphoglycerate dehydrogenase & 0.0606976 & 15 & Up \\
PRKD1 & Protein kinase D1 & 0.0550501 & 5 & Down \\
IQGAP1 & IQ motif containing GTPase activating & 0.0530205 & 11 & Down \\
protein 1 & Integrin, alpha 2 (CD49B, alpha 2 subunit & 0.0473438 & 20 & Down \\
ITGA2 & of VLA-2 receptor) & 0.0472054 & 16 & Up \\
TUBA4A & Tubulin, alpha 4a & 0.0441149 & 11 & Down \\
ANXA2 & Annexin A2 & 0.0385101 & 10 & Down \\
PFKP & Phosphofructokinase, platelet & 0.0365287 & 5 & Up \\
EPAS1 & Endothelial PAS domain protein 1 & 0.0286934 & 14 & Down \\
PLEC & Plectin & & Down \\
ALDH3A2 & Aldehyde dehydrogenase 3 family, member & 0.027284 & 11 & Down \\
ITGB3 & A2 & & Up \\
PLAT & Integrin, beta 3 (platelet glycoprotein IIIa, & 0.0205254 & 11 & Up \\
CDH1 & antigen CD61) & Plasminogen activator, tissue & 3 & Down \\
CABLES1 & Cadherin 1, type 1, E-cadherin (epithelial) & 0.0167665 & 4 & Up \\
LIN7A & Cdk5 and Abl enzyme substrate 1 & 0.0135026 & 11 & Down \\
AKAP9 & Lin-7 homolog A (C. elegans) & 0.0135026 & 4 & Down \\
ABCD3 & A kinase (PRKA) anchor protein (yotiao) 9 & 0.0135026 & 2 & 2 \\
\hline
\end{tabular}

scanner 3000 (Affymetrix). Data were analyzed using the Affymetrix Expression and Transcriptome Console Software. A random variance model $t$ test was used to filter the DE transcripts for experimental and control groups in the cases of small samples. After significance analysis and false discovery rate (FDR) analysis, the aberrantly expressed transcripts (lncRNAs, miRNAs, and mRNAs) were identified using $P$ values $(<0.05)$. The DE gene lists are shown in the Supplementary Dataset 1-3. The raw data for the microarray (.CEL) can be downloaded from Baidu SkyDrive (http://pan.baidu.com/ $\mathrm{s} / 1 \mathrm{pKNKoqf)}$.

\section{GO and KEGG pathway analyses}

The DE mRNAs and the predicted target genes of DE miRNAs were annotated in terms of their GO categories and KEGG pathways using the web-based tool, Database for Annotation, Visualization, and Integrated Discovery (http://david.abcc.ncifcrf.gov/). Fisher's exact test and $\chi^{2}$ test were used to select significant GO categories and KEGG pathways, and the threshold of significance was defined by $P<0.05$ (the FDR was used to correct the $P$ value).

\section{MiRNA target gene prediction and network construction}

Target genes of the DE miRNAs were predicted using the combined miRNA target prediction databases including miRanda (http://www.microrna.org/) and TargetScan (http://www.targetscan.org/). Only the target genes that were identified in both databases were considered further to reduce the false positivity. The predicted targets of deregulated miRNAs were compared with those of the deregulated mRNAs from the mRNA expression profile data to detect overlaps. The interactive network of miRNAs and actually deregulated target mRNAs was visualized using the Cytoscape software (http://www.cytoscape.org/). The miRNA-GO network was built according to the relationships between genes and significant GOs and the relationships among miRNAs and GOs.

\section{Gene signal transduction network and IncRNA- mRNA co-expression network}

The gene signal transduction network (signalnet) was constructed based on the data of DE genes. 
Table 2: Twenty-four genes with different pathway connectivities (identified with Diffk values) in patients with $\mathrm{AS}$ and controls $(|\mathrm{Diffk}| \geq 0.75)$

\begin{tabular}{lllll}
\hline Gene symbol & Description & Style & $\mid$ Diffk & Type \\
\hline SMYD5-AS2 & --- & down & 1 & noncoding \\
SLC2A1 & solute carrier family 2 (facilitated glucose & up & 0.875 & coding \\
& transporter), member 1 & & & \\
PDE4D & processed_transcript & down & 0.875 & noncoding \\
SLC9A9 & solute carrier family 9, subfamily A (NHE9, & down & 0.875 & coding \\
& cation proton antiporter 9), member 9 & & & \\
CSNK1D-AS8 & --- & up & 0.8125 & noncoding \\
SLC35F5 & solute carrier family 35, member F5 & down & 0.8125 & coding \\
BRD8-AS1 & --- & down & 0.8125 & noncoding \\
PCID2-AS7 & --- & down & 0.8125 & noncoding \\
REV1-AS3 & --- & down & 0.8125 & noncoding \\
OPN1SW-AS1 & --- & down & 0.8125 & noncoding \\
CHD1L-AS7 & --- & down & 0.8125 & noncoding \\
TARBP1-AS7 & --- & down & 0.8125 & noncoding \\
VDAC2 & retained_intron & down & 0.8125 & noncoding \\
REXO2-AS1 & --- & down & 0.75 & noncoding \\
NDRG1-AS6 & --- & up & 0.75 & noncoding \\
MFF-AS2 & --- & down & 0.75 & noncoding \\
WASHC4 & nonsense_mediated_decay & down & 0.75 & noncoding \\
SPINK5 & serine peptidase inhibitor, Kazal type 5 & up & 0.75 & coding \\
HSD3B7 & hydroxy-delta-5-steroid dehydrogenase, 3 beta- & down & 0.75 & coding \\
PRDM16 & and steroid delta-isomerase 7 & & & \\
CD46-AS9 & PR domain containing 16 & up & 0.75 & coding \\
RPLP0-AS3 & --- & down & 0.75 & noncoding \\
MGAT5-AS2 & --- & up & 0.75 & noncoding \\
VASH1 & vasohibin 1 & down & 0.75 & noncoding \\
& & down & 0.75 & coding \\
\hline
\end{tabular}

Table 3: Characteristics of patients with AS

\begin{tabular}{ll}
\hline Characteristics & Total sample $(\boldsymbol{N}=\mathbf{2 0})$ \\
\hline Age $($ year $)$ & $41.3 \pm 10.7$ \\
Sex $(\mathrm{M} / \mathrm{F})(n)$ & $19 / 1$ \\
BMI $\left(\mathrm{kg} / \mathrm{m}^{2}\right)$ & $20.5 \pm 1.6$ \\
Duration of AS (year) & $11.3 \pm 3.2$ \\
ESR $(\mathrm{mm} / \mathrm{h})$ & $15.6 \pm 11.0$ \\
CRP $(\mathrm{mg} / \mathrm{L})$ & $10.1 \pm 7.1$ \\
\hline
\end{tabular}

AS, Ankylosing spondylitis; BMI, body mass index; CRP, C-reactive protein; ESR, erythrocyte sedimentation rate.

The KEGG database was used to analyze functional gene interactions. In the network, nodes represented genes (protein, compound, and so forth) and edges represented relation types between the nodes (activation or phosphorylation, and so forth). The degree of a gene was defined as the number of directly linked genes within a network, which could evaluate the relative significance of a gene in the network. In the signal-net analysis, the genes were characterized by measuring their "betweenness centrality": the number of times a node was located in the shortest path between two other nodes. The CNC networks were constructed to identify interactions among genes and lncRNAs. They were built according to the normalized signal intensity of specific expression genes. 
In the present study, only lncRNA-mRNA pairs with the Pearson correlation coefficient (PCC) value $\geq 0.98$ and $P$ $<0.05$ were retained for network construction and further analysis. In considering different networks, the core regulatory genes were assessed by the degree of difference they showed in their roles in the AS and control networks, which was measured with the variable Diffk (difference in normalized connectivities). The co-expression networks were drawn using the Cytoscape software.

\section{Construction of the ceRNA co-expression network}

The ceRNA network was constructed based on the ceRNA theory as follows: (1) negatively correlated mRNA-miRNA pairs were screened, where the mRNAs were the targets of the miRNAs; (2) negatively correlated IncRNA-miRNA pairs were screened, where the lncRNAs were the targets of the miRNAs; (3) mRNA-lncRNA integrations were evaluated by screening mRNA-lncRNA pairs with positive co-expression relationships using PCC; and (4) both lncRNA and mRNA in an lncRNAmRNA pair were targeted and negatively correlated with a common miRNA. The lncRNA-miRNA-mRNA network was constructed by assembling all lncRNA-miRNAmRNA triplets, also known as ceRNAs.

\section{Cell culture and osteogenic differentiation}

Primary fibroblasts $(N=3)$ were isolated from hip ligament tissues using the previously described method with some modifications. The ligaments were cut into 0.3 -

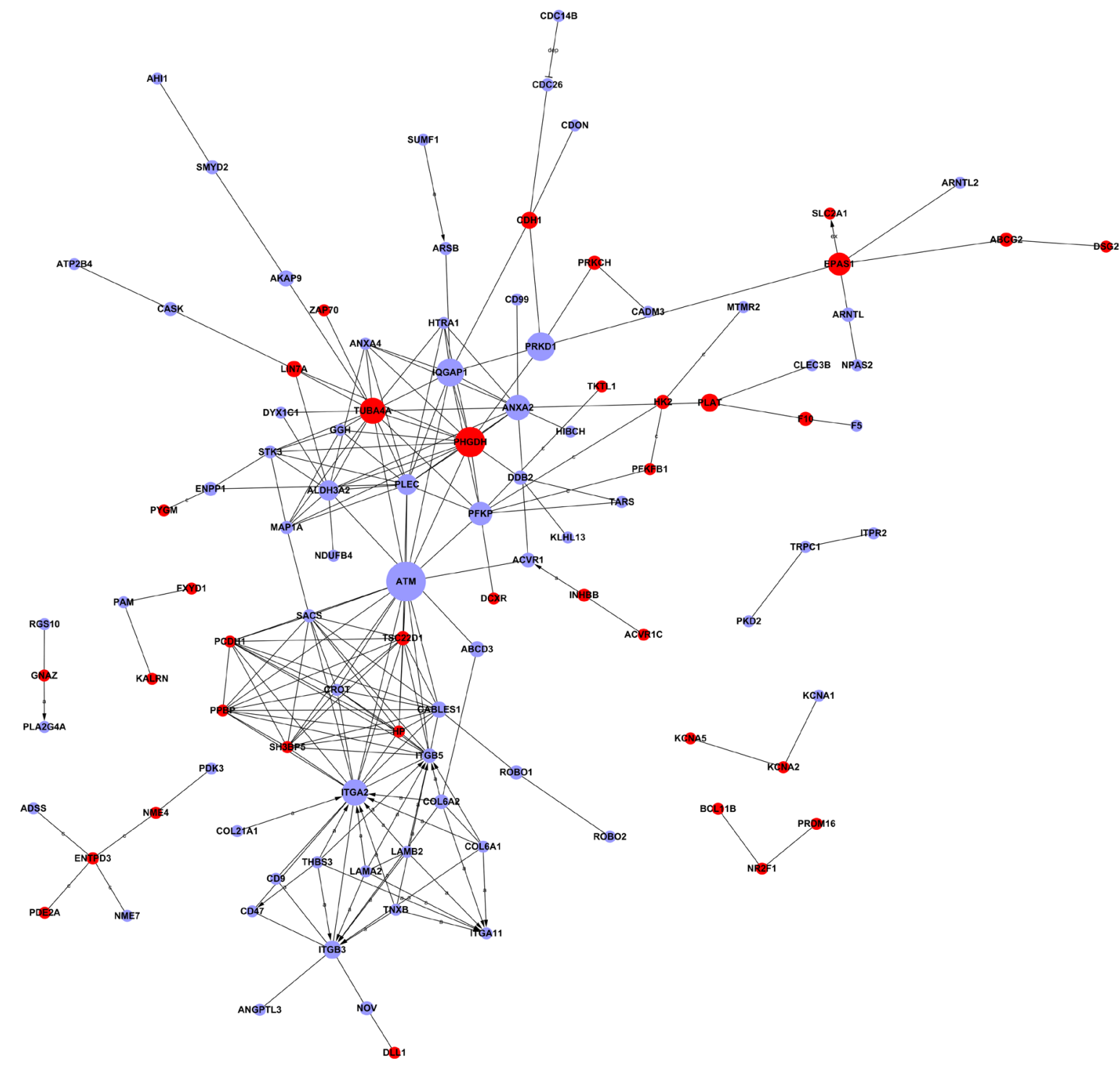

Figure 6: Interaction network of differentially expressed genes (signal-net). In the signal-net, the genes are characterized by measuring their "betweenness centrality": the number of times a node is located in the shortest path between two other nodes. This measure reflects the importance of a node in a network in relation to another. The circles represent important functional genes (red: upregulated genes; purple: downregulated genes); the circle size represents the degree of interaction (betweenness centrality), and lines indicate the interactions. 
$\mathrm{cm}^{3}$ pieces, washed twice with sterile phosphate-buffered saline (PBS) to remove blood, and centrifuged at 1500 revolutions per minute (rpm) for $5 \mathrm{~min}$. The ligaments were resuspended in fetal bovine serum (FBS; HyClone, GE Healthcare, Buckinghamshire, UK) and then seeded into $25-\mathrm{cm}^{2}$ flasks for $4 \mathrm{~h}$ at $37^{\circ} \mathrm{C}$. Then, FBS was replaced with Dulbecco's modified Eagle's medium (DMEM; HyClone) containing 15\% FBS, $100 \mathrm{U} / \mathrm{mL}$ penicillin, and $100 \mu \mathrm{g} / \mathrm{mL}$ streptomycin. After cells adhered, the ligament pieces were removed, and the culture medium was replaced every 3 days thereafter. The passages from 3 to 8 were used for all subsequent experiments. Flow cytometry (BD FACSCalibur, NJ, USA) was used for fibroblast identification marked by CD29 and CD90, as described in previous studies (Supplementary Figure 1). For osteogenic differentiation, the fibroblasts were seeded in 24-well plates in a growth medium consisting of DMEM with $10 \%$ FBS. When the culture reached a confluence of $80 \%-90 \%$, the medium was changed to OM containing $100 \mathrm{nM}$ dexamethasone, $10 \mathrm{mM} \beta$-glycerophosphate, 50 $\mu \mathrm{g} / \mathrm{mL}$ of ascorbic acid, and 10\% FBS. The fibroblasts were cultured in OM for up to 21 days, and the medium was replaced every 3 days.

\section{ALP activity and staining}

The ALP activity in the fibroblasts was detected using an ALP activity kit (Jiancheng Biotech, Nanjing,
China). Briefly, the fibroblasts were lysed in a lysis buffer containing $1 \%$ Triton X-100, 20mM Tris- $\mathrm{HCl}$ $(\mathrm{pH} 7.5)$, and $150 \mathrm{mM} \mathrm{NaCl}$. The lysate was centrifuged at $12,000 \mathrm{rpm}$ at $4^{\circ} \mathrm{C}$ for $5 \mathrm{~min}$, and the supernatant was incubated with reaction buffers at $37^{\circ} \mathrm{C}$ for $15 \mathrm{~min}$. Then, the coloration solution was added, and the absorbance was measured at $520 \mathrm{~nm}$. The total protein concentration was determined using a bicinchoninic acid protein assay kit (Pierce, MA, USA). The relative ALP activity was expressed as units per gram of protein per $15 \mathrm{~min}$. The ALP staining assay was performed using a BCIP/NBT ALP Color Development Kit (Beyotime, Shanghai, China). The fibroblasts were washed with PBS twice and fixed with $95 \%$ ethanol for $15 \mathrm{~min}$. The cells were washed with PBS twice and incubated with ALP dye solution in the dark for $30 \mathrm{~min}$. Then the reaction was stopped using distilled water, and the plate was dried before taking a photo.

\section{ARS staining and quantification}

Fibroblasts were washed with PBS and fixed with $95 \%$ ethanol for $15 \mathrm{~min}$. The cells were then stained with $1 \%$ ARS ( $\mathrm{pH} \mathrm{4.3)}$ for $15 \mathrm{~min}$ and washed three times with distilled water to remove nonspecific staining. The stained cells were visualized on photographs and observed under a microscope. For ARS quantification, the calcium deposition was destained with $10 \%$ cetylpyridinium

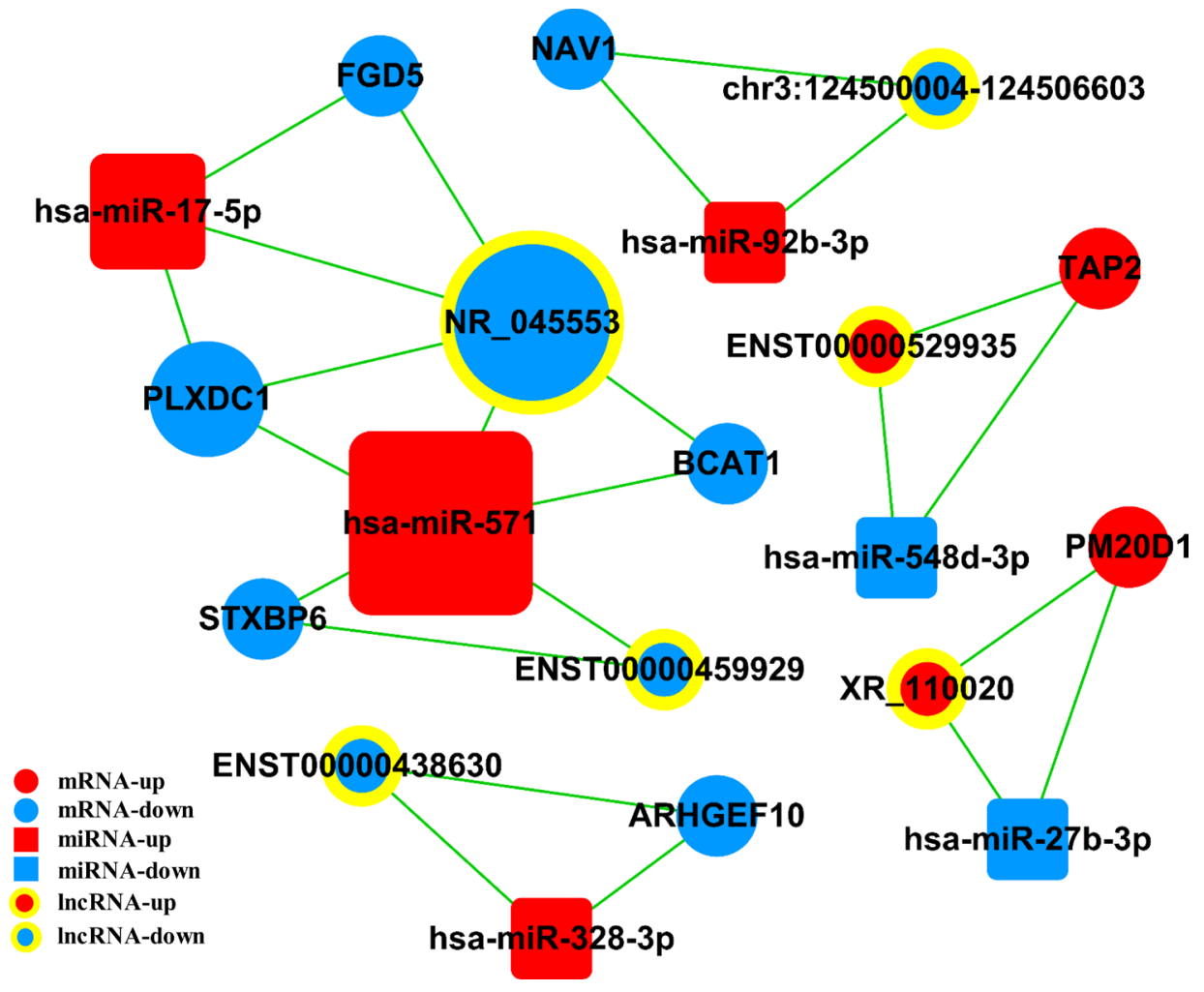

Figure 7: Competing endogenous RNA network in AS. The competing endogenous RNA network is based on miRNA-mRNA, miRNA-IncRNA, and lncRNA-mRNA interactions. Boxes represent miRNAs, circles represent mRNAs, and concentric circles represent lncRNAs. Red represents upregulated and blue represents downregulated genes. AS, Ankylosing spondylitis. 

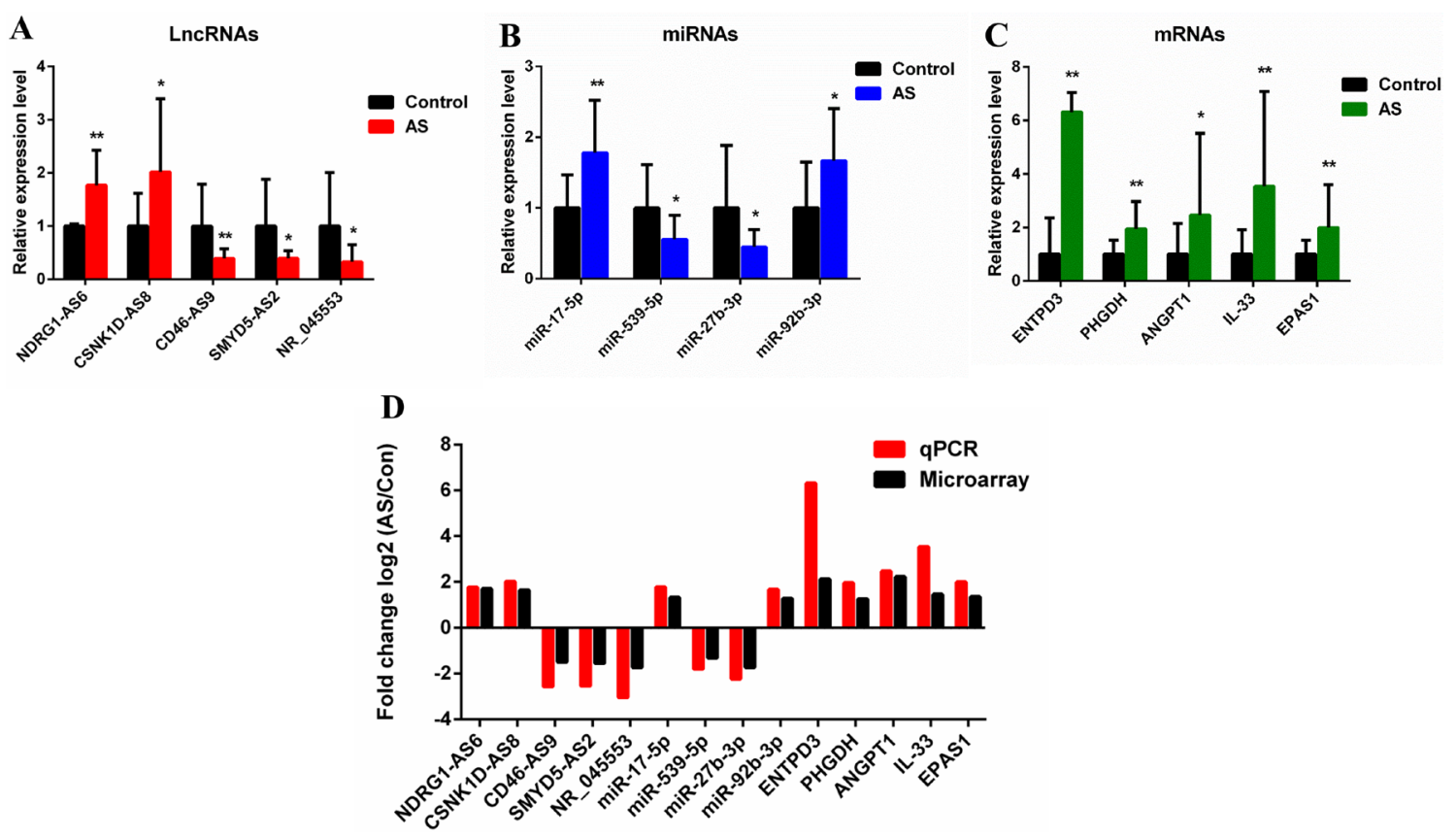

Figure 8: Validation of microarray data by qRT-PCR. (A) Five lncRNAs, (B) four miRNAs, and (C) five mRNAs were validated by qRT-PCR between AS and control groups. The relative expression level of each RNA was normalized. The data displayed in histograms are expressed as means \pm standard deviation. ${ }^{*} P<0.05 ;{ }^{* *} P<0.01$ compared with the control group. (D) Comparison between qRT-PCR results and microarray data revealing a good correlation between the two methods. The heights of the columns represent the fold changes ( $\log 2$ transformed) computed using the qRT-PCR and microarray data. qRT-PCR, Quantitative real-time polymerase chain reaction.
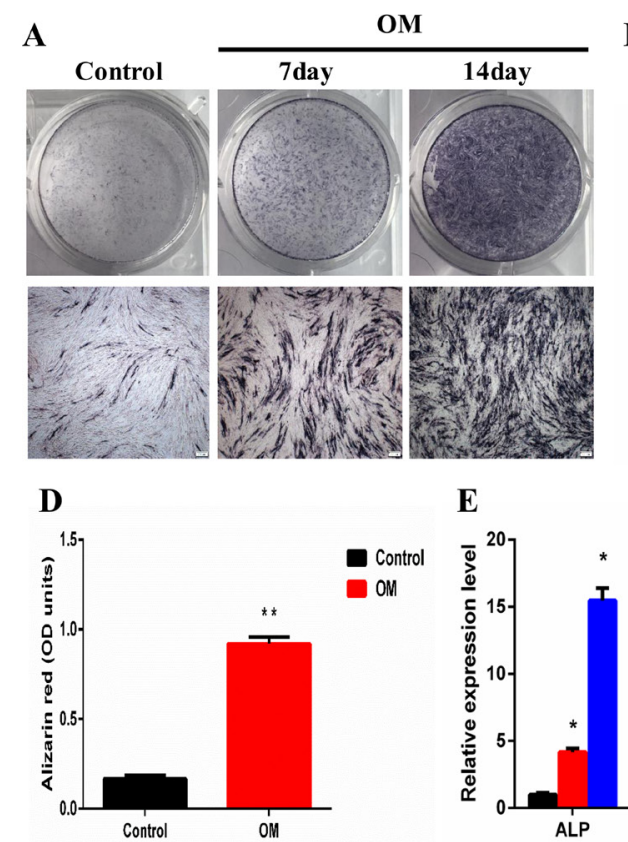

B

$\mathbf{E}$

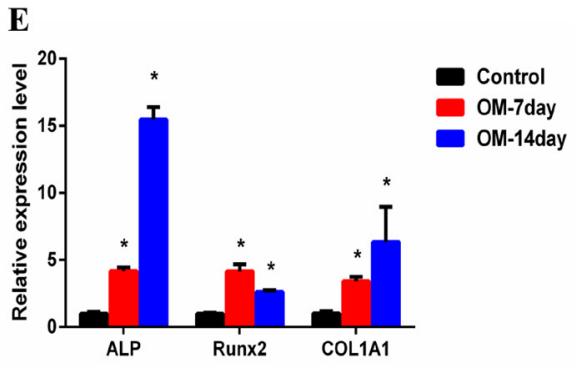

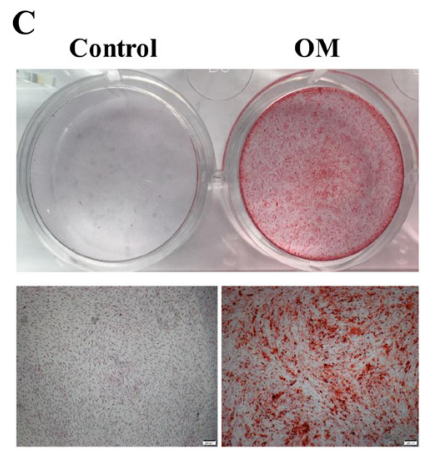

F

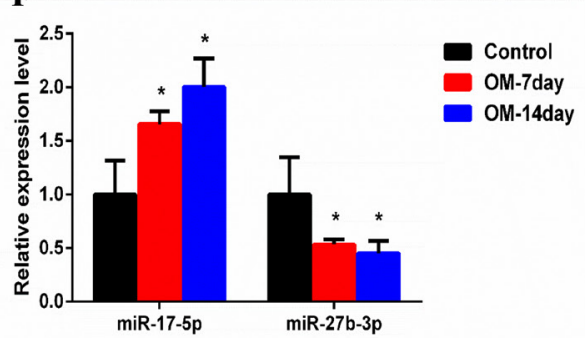

Figure 9: MiR-17-5p and miR-27b-3p were differentially expressed during osteogenic differentiation of human ligament fibroblasts. (A) ALP in fibroblasts was stained using the BCIP/NBT kit after the cells were incubated in OM for 7 and 14 days. Scale bar $=200 \mu \mathrm{m}$. (B) The ALP activity of fibroblasts was measured after they were incubated in OM for 7 and 14 days. (C) Fibroblasts were incubated in OM for 21 days, and then the mineralized nodules were stained using alizarin red S (ARS). Scale bar $=200 \mu \mathrm{m}$. (D) Mineralization was quantified by extraction of ARS dye with $10 \%$ cetylpyridinium chloride. (E) The total RNA was isolated on days 7 and 14. Runx2, ALP, and COL1A1 mRNA levels were determined using qRT-PCR and normalized to GAPDH. (F) The total RNA was isolated on days 7 and 14. Levels of $m i R-17-5 p$ and $m i R-27 b-3 p$ were determined using qRT-PCR and normalized to U6. ${ }^{*} P<0.05 ;{ }^{* *} P<0.01$ compared with controls. ALP, Alkaline phosphatase; GADPH, glyceraldehyde-3-phosphate dehydrogenase; OM, osteogenic differentiation medium; qRT-PCR, Quantitative real-time polymerase chain reaction. 
chloride (Sigma-Aldrich, MO, USA) in $10 \mathrm{mM}$ sodium phosphate for $1 \mathrm{~h}$ at room temperature. The absorbance was measured using a microplate reader at $562 \mathrm{~nm}$.

\section{qRT-PCR}

The total RNA was isolated with a TRIzol reagent (Invitrogen) according to the manufacturer's instructions. For lncRNAs and mRNAs, the total RNA was reverse transcribed into cDNA using a PrimeScript RT reagent kit (Takara, Japan). Ordinary qRT-PCR was performed on a LightCycler 480 PCR system (Roche, Germany) using SYBR Premix Ex Taq II (Takara, Japan). MiRNA qRT-PCR was performed using a Bulge-Loop miRNA qRT-PCR Starter Kit (RiboBio, Guangzhou, China). The lncRNA/mRNA and miRNA PCR results were quantified using the $2^{-\Delta \Delta \mathrm{Ct}}$ method, with normalization using glyceraldehyde-3-phosphate dehydrogenase (GAPDH) and U6, respectively. Fold changes were shown as means \pm standard deviation (SD) from three independent experiments. The primer sets specific for miRNA and IncRNA were purchased from Ribobio (Guangzhou, China). The forward and reverse primers for some genes are presented in Supplementary Table 1.

\section{Statistical analysis}

The statistical analysis was performed using the SPSS 19.0 software (SPSS Inc., IL, USA). All data were expressed as mean $\pm \mathrm{SD}$. The Student $t$ test was performed to analyze the microarray and qRT-PCR data. A $P$ value $<$ 0.05 was considered statistically significant.

\section{Abbreviations}

AS: Ankylosing spondylitis; lncRNA: long noncoding RNA; miRNA: microRNA; mRNA: messenger RNA; ceRNA: competing endogenous RNA; ncRNA: noncoding RNA; DE: differentially expressed; OA: osteoarthritis; RA: rheumatoid arthritis; GO: Gene Ontology; KEGG: Kyoto Encyclopedia of Genes and Genomes; CNC: coding-noncoding gene coexpression; qRT-PCR: quantitative real-time polymerase chain reaction; OM: osteogenic differentiation medium; PCC: Pearson correlation coefficient; ALP: alkaline phosphatase; ARS: alizarin red S.

\section{Author contributions}

Chen Zhang, Chen Wang, Zhenyu Jia, Xuan Huang, and Weidong Xu conceived and designed the experiments. Chen Zhang, Chen Wang, Zhenyu Jia, Xuan Huang, and Weidong Xu performed the experiments. Chen Zhang, Chen Wang, Xuan Huang, and Weidong $\mathrm{Xu}$ wrote the paper and prepared the figures. Delin Liu, Wenwen Tong, and Chongru He contributed reagents/materials/analysis tools. All authors read and approved the manuscript.

\section{ACKNOWLEDGMENTS AND FUNDING}

The authors thank all the patients who participated in the study. This work was supported by the National Natural Science Foundation of China (Grant No. 81672126 and No.81601412).

\section{CONFLICTS OF INTEREST}

The authors declare no conflicts of interest.

\section{REFERENCES}

1. Taurog JD, Chhabra A, Colbert RA. Ankylosing Spondylitis and Axial Spondyloarthritis. N Engl J Med. 2016; 374:2563-2574.

2. Braun J, Sieper J. Ankylosing spondylitis. Lancet. 2007; 369:1379-1390.

3. Rashid T, Ebringer A. Ankylosing spondylitis is linked to Klebsiella--the evidence. Clin Rheumatol. 2007; 26:858864.

4. Lou YJ, Jin J, Mai WY. Ankylosing spondylitis presenting with macrophage activation syndrome. Clin Rheumatol. 2007; 26:1929-1930.

5. Braun J, Bollow M, Neure L, Seipelt E, Seyrekbasan F, Herbst H, Eggens U, Distler A, Sieper J. Use of immunohistologic and in situ hybridization techniques in the examination of sacroiliac joint biopsy specimens from patients with ankylosing spondylitis. Arthritis Rheum. 1995; 38:499-505.

6. Appel H, Maier R, Bleil J, Hempfing A, Loddenkemper C, Schlichting U, Syrbe U, Sieper J. In situ analysis of interleukin-23- and interleukin-12-positive cells in the spine of patients with ankylosing spondylitis. Arthritis Rheum. 2013; 65:1522-1529.

7. Appel H, Maier R, Wu P, Scheer R, Hempfing A, Kayser R, Thiel A, Radbruch A, Loddenkemper C, Sieper J. Analysis of IL-17(+) cells in facet joints of patients with spondyloarthritis suggests that the innate immune pathway might be of greater relevance than the Th17-mediated adaptive immune response. Arthritis Res Ther. 2011; 13:R95.

8. Ciccia F, Accardo-Palumbo A, Alessandro R, Rizzo A, Principe S, Peralta S, Raiata F, Giardina A, De Leo G, Triolo G. Interleukin-22 and interleukin-22-producing NKp44+ natural killer cells in subclinical gut inflammation in ankylosing spondylitis. Arthritis Rheum. 2012; 64:18691878.

9. Ciccia F, Guggino G, Rizzo A, Saieva L, Peralta S, Giardina A, Cannizzaro A, Sireci G, De Leo G, Alessandro R, Triolo G. Type 3 innate lymphoid cells producing IL-17 and IL-22 are expanded in the gut, in the peripheral blood, synovial fluid and bone marrow of patients with ankylosing spondylitis. Ann Rheum Dis. 2015; 74:1739-1747. 
10. Colbert RA, Tran TM, Layh-Schmitt G. HLA-B27 misfolding and ankylosing spondylitis. Mol Immunol. 2014; $57: 44-51$.

11. Ciccia F, Accardo-Palumbo A, Rizzo A, Guggino G, Raimondo S, Giardina A, Cannizzaro A, Colbert RA, Alessandro R, Triolo G. Evidence that autophagy, but not the unfolded protein response, regulates the expression of IL-23 in the gut of patients with ankylosing spondylitis and subclinical gut inflammation. Ann Rheum Dis. 2014; 73:1566-1574.

12. Ciccia F, Haroon N. Autophagy in the pathogenesis of ankylosing spondylitis. Clin Rheumatol. 2016; 35:14331436.

13. Kim TJ, Kim TH, Jun JB, Joo KB, Uhm WS. Prevalence of ossification of posterior longitudinal ligament in patients with ankylosing spondylitis. J Rheumatol. 2007; 34:2460-2462.

14. Benjamin M, McGonagle D. Entheses: tendon and ligament attachment sites. Scand J Med Sci Sports. 2009; 19:520-527.

15. Gillet P, Pere P, Jouzeau JY, Floquet J, Gaucher A. Enthesitis of the ligamentum teres during ankylosing spondylitis: histopathological report. Ann Rheum Dis. 1994; 53:82.

16. Yu F, Cui Y, Zhou X, Zhang X, Han J. Osteogenic differentiation of human ligament fibroblasts induced by conditioned medium of osteoclast-like cells. Biosci Trends. 2011; 5:46-51.

17. Rutherford RB, Moalli M, Franceschi RT, Wang D, Gu K, Krebsbach PH. Bone morphogenetic protein-transduced human fibroblasts convert to osteoblasts and form bone in vivo. Tissue Eng. 2002; 8:441-452.

18. Yang M, Yuan H, Miao M, Xu W. The osteogenic potential of ligament fibroblasts is greater in ankylosing spondylitis patients than in patients with osteoarthritis. Z Rheumatol. 2015; 74:340-345.

19. Bartel DP. MicroRNAs: genomics, biogenesis, mechanism, and function. Cell. 2004; 116:281-297.

20. Chen L, Al-Mossawi MH, Ridley A, Sekine T, Hammitzsch A, de Wit J, Simone D, Shi H, Penkava F, KurowskaStolarska M, Pulyakhina I, Knight JC, Kim TJ, et al. miR10b-5p is a novel Th17 regulator present in Th17 cells from ankylosing spondylitis. Ann Rheum Dis. 2017; 76:620-625.

21. Qian BP, Ji ML, Qiu Y, Wang B, Yu Y, Shi W, Luo YF. Identification of Serum miR-146a and miR-155 as Novel Noninvasive Complementary Biomarkers for Ankylosing Spondylitis. Spine (Phila Pa 1976). 2016; 41:735-742.

22. Huang J, Song G, Yin Z, Luo X, Ye Z. Elevated miR-29a expression is not correlated with disease activity index in PBMCs of patients with ankylosing spondylitis. Mod Rheumatol. 2013 Apr 30. [Epub ahead of print].

23. Mercer TR, Dinger ME, Mattick JS. Long non-coding RNAs: insights into functions. Nat Rev Genet. 2009; 10:155-159.

24. Zhang Y, Xu YZ, Sun N, Liu JH, Chen FF, Guan XL, Li A, Wang F, Zhao QF, Wang HY, Song SS, Yu W, Zhao JN, et al. Long noncoding RNA expression profile in fibroblast- like synoviocytes from patients with rheumatoid arthritis. Arthritis Res Ther. 2016; 18:227.

25. Fu M, Huang G, Zhang Z, Liu J, Zhang Z, Huang Z, Yu B, Meng F. Expression profile of long noncoding RNAs in cartilage from knee osteoarthritis patients. Osteoarthritis Cartilage. 2015; 23:423-432.

26. Salmena L, Poliseno L, Tay Y, Kats L, Pandolfi PP. A ceRNA hypothesis: the Rosetta Stone of a hidden RNA language? Cell. 2011; 146:353-358.

27. Zhou M, Wang X, Shi H, Cheng L, Wang Z, Zhao H, Yang L, Sun J. Characterization of long non-coding RNA-associated ceRNA network to reveal potential prognostic lncRNA biomarkers in human ovarian cancer. Oncotarget. 2016; 7:12598-12611. https://doi.org/10.18632/oncotarget.7181.

28. Yang S, Ning Q, Zhang G, Sun H, Wang Z, Li Y. Construction of differential mRNA-lncRNA crosstalk networks based on ceRNA hypothesis uncover key roles of IncRNAs implicated in esophageal squamous cell carcinoma. Oncotarget. 2016; 7:85728-85740. https://doi. org/10.18632/oncotarget.13828.

29. Wei C, Luo T, Zou S, Zhou X, Shen W, Ji X, Li Q, Wu A. Differentially expressed lncRNAs and miRNAs with associated ceRNA networks in aged mice with postoperative cognitive dysfunction. Oncotarget. 2017; 8:55901-55914. https://doi.org/10.18632/oncotarget.18362.

30. Lai K, Jia S, Yu S, Luo J, He Y. Genome-wide analysis of aberrantly expressed lncRNAs and miRNAs with associated co-expression and ceRNA networks in beta-thalassemia and hereditary persistence of fetal hemoglobin. Oncotarget. 2017; 8:49931-49943. https://doi.org/10.18632/oncotarget.18263.

31. Jiang H, Ma R, Zou S, Wang Y, Li Z, Li W. Reconstruction and analysis of the lncRNA-miRNA-mRNA network based on competitive endogenous RNA reveal functional lncRNAs in rheumatoid arthritis. Mol Biosyst. 2017; 13:1182-1192.

32. Brown MA, Kenna T, Wordsworth BP. Genetics of ankylosing spondylitis--insights into pathogenesis. Nat Rev Rheumatol. 2016; 12:81-91.

33. Xie Z, Li J, Wang P, Li Y, Wu X, Wang S, Su H, Deng W, Liu Z, Cen S, Ouyang Y, Wu Y, Shen H. Differential Expression Profiles of Long Noncoding RNA and mRNA of Osteogenically Differentiated Mesenchymal Stem Cells in Ankylosing Spondylitis. J Rheumatol. 2016; 43:1523-1531.

34. Xie Z, Wang P, Li Y, Deng W, Zhang X, Su H, Li D, Wu Y, Shen H. Imbalance Between Bone Morphogenetic Protein 2 and Noggin Induces Abnormal Osteogenic Differentiation of Mesenchymal Stem Cells in Ankylosing Spondylitis. Arthritis Rheumatol. 2016; 68:430-440.

35. Qin X, Jiang T, Liu S, Tan J, Wu H, Zheng L, Zhao J. Effect of metformin on ossification and inflammation of fibroblasts in ankylosing spondylitis: An in vitro study. J Cell Biochem. 2018; 119:1074-1082.

36. Raychaudhuri SK, Raychaudhuri SP. Janus kinase/signal transducer and activator of transcription pathways in spondyloarthritis. Curr Opin Rheumatol. 2017; 29:311-316. 
37. Chen C, Zhang X, Wang Y. Analysis of JAK2 and STAT3 polymorphisms in patients with ankylosing spondylitis in Chinese Han population. Clin Immunol. 2010; 136:442446.

38. Tang S, Hou Y, Zhang H, Tu G, Yang L, Sun Y, Lang L, Tang X, Du YE, Zhou M, Yu T, Xu L, Wen S, et al. Oxidized ATM promotes abnormal proliferation of breast CAFs through maintaining intracellular redox homeostasis and activating the PI3K-AKT, MEK-ERK, and Wnt-betacatenin signaling pathways. Cell Cycle. 2015; 14:19081924.

39. Peters MA, Wendholt D, Strietholt S, Frank S, Pundt N, Korb-Pap A, Joosten LA, van den Berg WB, Kollias G, Eckes B, Pap T. The loss of alpha2beta1 integrin suppresses joint inflammation and cartilage destruction in mouse models of rheumatoid arthritis. Arthritis Rheum. 2012; 64:1359-1368.

40. Drouart M, Saas P, Billot M, Cedoz JP, Tiberghien P, Wendling D, Toussirot E. High serum vascular endothelial growth factor correlates with disease activity of spondylarthropathies. Clin Exp Immunol. 2003; 132:158 162.

41. Raghunath M, Tontsidou L, Oji V, Aufenvenne K, Schurmeyer-Horst F, Jayakumar A, Stander H, Smolle J, Clayman GL, Traupe H. SPINK5 and Netherton syndrome: novel mutations, demonstration of missing LEKTI, and differential expression of transglutaminases. J Invest Dermatol. 2004; 123:474-483.

42. Jiang J. CK1 in Developmental Signaling: Hedgehog and Wnt. Curr Top Dev Biol. 2017; 123:303-329.

43. Greer YE, Rubin JS. Casein kinase 1 delta functions at the centrosome to mediate Wnt-3a-dependent neurite outgrowth. J Cell Biol. 2011; 192:993-1004.

44. Hosoi F, Izumi H, Kawahara A, Murakami Y, Kinoshita H, Kage M, Nishio K, Kohno K, Kuwano M, Ono M. N-myc downstream regulated gene 1/Cap43 suppresses tumor growth and angiogenesis of pancreatic cancer through attenuation of inhibitor of kappaB kinase beta expression. Cancer Res. 2009; 69:4983-4991.

45. Tsai YG, Niu DM, Yang KD, Hung CH, Yeh YJ, Lee CY, Lin CY. Functional defects of CD46-induced regulatory $\mathrm{T}$ cells to suppress airway inflammation in mite allergic asthma. Lab Invest. 2012; 92:1260-1269.
46. Burney RO, Pile KD, Gibson K, Calin A, Kennedy LG, Sinnott PJ, Powis SH, Wordsworth BP. Analysis of the MHC class II encoded components of the HLA class I antigen processing pathway in ankylosing spondylitis. Ann Rheum Dis. 1994; 53:58-60.

47. Qian Y, Wang G, Xue F, Chen L, Wang Y, Tang L, Yang H. Genetic association between TAP1 and TAP2 polymorphisms and ankylosing spondylitis: a systematic review and meta-analysis. Inflamm Res. 2017; 66:653-661.

48. Krintel SB, Essioux L, Wool A, Johansen JS, Schreiber E, Zekharya T, Akiva P, Ostergaard M, Hetland ML. CD6 and syntaxin binding protein 6 variants and response to tumor necrosis factor alpha inhibitors in Danish patients with rheumatoid arthritis. PLoS One. 2012; 7:e38539.

49. Murata K, Yoshitomi H, Tanida S, Ishikawa M, Nishitani K, Ito H, Nakamura T. Plasma and synovial fluid microRNAs as potential biomarkers of rheumatoid arthritis and osteoarthritis. Arthritis Res Ther. 2010; 12:R86.

50. Fang T, Wu Q, Zhou L, Mu S, Fu Q. miR-106b-5p and miR-17-5p suppress osteogenic differentiation by targeting Smad5 and inhibit bone formation. Exp Cell Res. 2016; 347:74-82.

51. Li H, Li T, Wang S, Wei J, Fan J, Li J, Han Q, Liao L, Shao C, Zhao RC. miR-17-5p and miR-106a are involved in the balance between osteogenic and adipogenic differentiation of adipose-derived mesenchymal stem cells. Stem Cell Res. 2013; 10:313-324.

52. Wei B, Wei W, Zhao B, Guo X, Liu S. Long noncoding RNA HOTAIR inhibits miR-17-5p to regulate osteogenic differentiation and proliferation in nontraumatic osteonecrosis of femoral head. PLoS One. 2017; 12:e169097.

53. Peng W, Zhu S, Li X, Weng J, Chen S. miR-27b-3p Suppressed Osteogenic Differentiation of Maxillary Sinus Membrane Stem Cells by Targeting Sp7. Implant Dent. 2017; 26:492-499.

54. van der Linden S, Valkenburg HA, Cats A. Evaluation of diagnostic criteria for ankylosing spondylitis. A proposal for modification of the New York criteria. Arthritis Rheum. $1984 ; 27: 361-368$. 